\title{
Medidas de distanciamento social e demandas para reorganização dos serviços hemoterápicos no contexto da Covid-19
}

\author{
Social distancing measures and demands for the reorganization \\ of hemotherapy services in the context of Covid-19
}

Mariluce Karla Bomfim de Souza (https://orcid.org/0000-0002-7895-4432) ${ }^{1}$

${ }^{1}$ Instituto de Saúde Coletiva, Universidade Federal da Bahia. R. Basílio da Gama s/n, Canela. 40110-040 Salvador BA Brasil.

marilucejbv@yahoo.com.br

\begin{abstract}
The article aims to discuss the consequences of social distancing measures on the availability of blood and organization of blood therapy services at the beginning of the Covid-19 pandemic in Brazil. News published in April 2020 on the websites of the country's state Blood Service Networks were consulted and organized in an Excel spreadsheet, presented in summary charts, and descriptions of results were prepared. A critical situation of blood supply, especially of some blood types, has been observed in many states. This situation is influenced by the circulation of the new coronavirus. The adoption of social distancing measures associated with unchanged transfusion demands for outpatient, urgency and emergency care required the implementation of strategies and actions for the reorganization of the services. Protection measures were incorporated, flows were changed and new routines were established. This study shows the extent to which the epidemiological situation of Covid-19 and the necessary measures for its control influenced the stocks and availability of blood. Changes in the organization of blood therapy services were fundamental in order to ensure protection, mitigate the risks of spreading the virus, and ensure the blood supply to meet the needs of the health system.
\end{abstract}

Key words Coronavirus infections, Brazil, Blood Therapy Service, Organization and administration
Resumo O artigo tem por objetivo discutir as consequências das medidas de distanciamento social sobre a disponibilidade de sangue e a organização dos serviços hemoterápicos no início da pandemia de Covid-19 no Brasil. A partir do acesso às notícias publicadas no mês de abril de 2020 nos sites das Hemorredes estaduais do país e organizadas em matriz Excel, foram elaborados quadros-sintese e descrição de resultados. A situação crítica de abastecimento de sangue, em especial para alguns tipos sanguíneos, foi constatada em muitos estados, devido à circulação do novo coronavírus. A adoção de medidas de distanciamento social e a manutenção das demandas por transfusão para tratamentos, urgência e emergência, requereram a implantação e a implementação de estratégias e ações para reorganização dos serviços. Medidas de proteção foram incorporadas, fluxos alterados e rotinas estabelecidas. Este estudo evidencia o quanto a situação epidemiológica da Covid-19 e as medidas necessárias para o seu controle influenciaram sobre a situação dos estoques e disponibilidade de sangue, e, demandaram, mudanças na organização dos serviços hemoterápicos a fim de assegurar a proteção, mitigar os riscos de propagação do vírus e garantir o suprimento de sangue para o atendimento às necessidades do sistema de saúde.

Palavras-chave Infecções por coronavírus, Brasil, Serviço de Hemoterapia, Organização e administração 


\section{Introdução}

Estudos apontam que a disseminação do vírus SARS-Cov-2, agente etiológico da doença conhecida como Covid-19, trouxe impacto no número de doações de sangue e, portanto, no suprimento de produtos sanguíneos aos serviços de saúde, além de aquecer a discussão sobre a segurança do sangue e medidas de vigilância, ainda que não exista evidência científica comprovada de transmissão do vírus por transfusão ${ }^{1-3}$.

$\mathrm{O}$ aumento do número de casos confirmados de SARS-Cov-2 tem levado os governos a decidirem pela adoção de medidas de distanciamento social com interrupção das atividades na comunidade, pela necessidade do achatamento da curva, o que coloca desafios para os serviços de saúde, inclusive para os serviços hemoterápicos, cujo recurso terapêutico que oferta depende da disponibilidade e voluntariedade do doador, além de ser um produto perecível ${ }^{4}$ e que necessita de constante reabastecimento ${ }^{5}$.

A adoção de medidas de distanciamento social tem por objetivo a redução das interações em uma comunidade, considerando que podem existir pessoas infectadas ainda não identificadas e, inclusive, não isoladas. Nesse sentido, a menor circulação e a não aglomeração de pessoas permite reduzir a transmissão de doenças cujo contágio se dá pela proximidade física das pessoas ${ }^{6}$.

São exemplos de medidas que têm por finalidade o distanciamento social, o fechamento de escolas e de locais de trabalho, a suspensão de alguns tipos de comércio e o cancelamento de eventos/reuniões para evitar aglomeração de pessoas $^{6}$. Tais iniciativas trouxeram impacto na oferta de "recursos inestimáveis" à saúde, de modo que o surgimento da pandemia levou a interrupção de atividades na comunidade, o fechamento de centros/unidades de doação em muitas áreas ao redor do mundo, atingindo o ponto em que a demanda ultrapassa o suprimento ${ }^{4}$.

Portanto, a adoção das medidas para evitar a propagação do vírus aplicado por muitos países à população global ameaça a oferta do sangue, por exemplo, exclusões por precaução, quarentena ou confinamento e temores de causar auto-exclusões $^{4,7}$. Ainda, a incerteza, o medo, as suposições existentes na mente de doadores e a relutância de ir aos serviços para doar sangue implicam na redução significativa do quantitativo de sangue coletado globalmente ${ }^{8}$.

Em uma publicação recente, responsáveis por bancos de sangue e departamentos de transfusão em hospitais de 12 países do mundo, incluindo
Austrália, Brasil, Canadá, Dinamarca, Irã, Israel, Itália, Japão, Coreia, Espanha, Reino Unido e Estados Unidos, informaram sobre as diferentes expectativas e prazos para a escassez de sangue considerando os níveis da pandemia nos seus países registrados até a penúltima semana do mês de abril. Os entrevistados israelenses, espanhóis, dinamarqueses, britânicos e coreanos não esperavam uma escassez de sangue como resultado dessa pandemia, enquanto que os demais esperavam escassez a curto, médio ou longo prazo 9 .

Portanto, a situação de pandemia e a adoção de medidas como distanciamento físico e bloqueio de cidades, províncias ou países na tentativa de conter a propagação da infecção podem resultar em um declínio maior no suprimento de sangue e na escassez geral de produtos sanguíneos ${ }^{10}$, cabendo destacar que os pacientes que sofrem de câncer, distúrbios hematológicos, bem como, os serviços de cirurgia, urgência e emergência precisam que os componentes do sangue sejam disponibilizados e prescritos.

Nesse sentido, este artigo tem por objetivo discutir as consequências das medidas de distanciamento social sobre a disponibilidade de sangue e a organização dos serviços hemoterápicos no início da pandemia de Covid-19 no Brasil.

\section{Metodologia}

Com o intuito de acompanhar as consequências da pandemia e as estratégias adotadas pelos serviços hemoterápicos para assegurar a disponibilidade de sangue para o atendimento às demandas dos serviços de saúde, foi construído um banco de notícias em Excel a partir dos sites das Hemorredes estaduais do Brasil. A primeira síntese produzida com base em tais notícias foi publicada na página do Observatório de Análise Política em Saúde (https://www.analisepoliticaemsaude. org/oaps $/)^{11}$, considerando o período de 26 de fevereiro (data de notificação do primeiro caso confirmado no Brasil, no estado de São Paulo) até o dia 31 de março de 2020.

Em 31 de março de 2020, registravam-se 5.824 casos confirmados de Covid-19 no Brasil, e tal registro saltou para 87.126 casos confirmados em 30 de abril de 2020 (https://painel.covid19br.org/). Nesse sentido, este estudo apresenta a seguinte questão: de que modo as medidas de distanciamento social adotadas influenciaram os estoques de sangue e sobre a organização e funcionamento dos serviços hemoterápicos nos primeiros meses da pandemia de Covid-19 no Brasil? 
As notícias publicadas nos sites das Hemorredes estaduais do Brasil e tomadas para discussão neste artigo contemplam as publicações do mês de abril do ano de 2020 e estão sumarizadas em dois quadros de resultados.

A discussão dos resultados segue cotejada com a síntese de experiências e publicações de outros países, identificadas a partir de levantamento no PubMed (a partir dos descritores: Covid-19 or novel coronavirus or coronavirus disease 2019 and blood transfusion or blood donors or blood supply or blood bank or blood donation or transfusion medicine), enquanto uma das atividades desenvolvidas pela autora na Rede Covida - Ciência, Informação e Solidariedade, a qual caracteriza-se como um projeto de colaboração científica e multidisciplinar focado na pandemia de Covid-19 resultado da parceria entre o Centro de Integração de Dados e Conhecimentos para Saúde (Cidacs/Fiocruz Bahia) e a Universidade Federal da Bahia (UFBA) (https://covid19br. org/).

\section{Resultados}

Os registros apontam que a pandemia de Covid-19 provocou mudanças na organização e funcionamento dos serviços hemoterápicos, inclusive com redução nos níveis de estoques de sangue, dadas as medidas de distanciamento social necessariamente adotadas para controlar a propagação e a transmissão do vírus. Nesse sentido, o Quadro 1 sumariza tais resultados.

Conforme as notícias publicadas nos sites das Hemorredes estaduais no mês de abril de 2020, constata-se "situação crítica" de abastecimento, em especial, para os tipos sanguíneos com fator $\mathrm{RH}$ negativo, $\mathrm{O}$ positivo e plaquetas, sendo que em alguns estados a redução foi registrada, no início do mês, em 30\% do número de doações diárias (Centro de Hematologia e Hemoterapia de Alagoas - Hemoal, 07.04.20) ou 35\% em seu número total de doadores (Instituto Estadual de Hematologia Arthur de Siqueira Cavalcanti do Rio de Janeiro - Hemorio, 07.04.20) até pelo menos 60\% dos doadores (Centro de Hemoterapia e Hematologia do Amazonas - Hemoam, 01.04.20).

Tal situação, apresentada nos sites, segue justificada/comprometida pela "pandemia do novo coronavírus (Covid-19)” (Centro de Hematologia e Hemoterapia do Maranhão, 03 e 08.04.20 Hemomar; Fundação de Hematologia e Hemoterapia de Rondônia - Fhemeron, 24.04.20) e pela expansão do número de casos no Brasil, segundo notícia da Fundação Hospitalar de Hematologia e Hemoterapia do Amazonas (Hemoam).

Conforme posicionamentos identificados nos sites, diretores e responsáveis pela captação e demais departamentos do ciclo do sangue declararam preocupação com a situação. Sobre isso a diretora da Fundação Hemoam destacou que "mesmo com o Hemocentro adotando medidas para evitar aglomerações de pessoas, agendamento, alteração do layout das salas de espera para manter uma distância mínima entre os doadores, e colocando dispensadores de álcool em gel à vontade, as doações são cada vez mais reduzidas" e, acrescentou que "a pandemia desacelerou o funcionamento de tudo", alertando que "o consumo dos produtos sanguíneos ainda é alto" (Hemoam, 01.04.20).

Informação do site do Centro de Hematologia e Hemoterapia do Maranhão (Hemomar) destaca que "por conta das medidas de isolamento social adotadas para combater a pandemia de Covid-19, as doações de sangue tiveram uma queda e os estoques de bolsas ficaram em nível crítico" (Hemomar, 08.04.20). Sobre isso, no site da Fundação Centro de Hematologia e Hemoterapia de Minas Gerais, reafirma-se que neste momento de isolamento social, "reflexos podem ser observados no fluxo de doadores" (Fundação Centro de Hematologia e Hemoterapia de Minas Gerais - Hemominas, 15.04.20).

Também foi enfatizada em algumas publicações que a necessidade de sangue se mantém, casos estes que se devem, inclusive, a pacientes com anemia falciforme e talassemias (Fhemeron, 02.04.20). A necessidade de sangue e hemocomponentes para tais atendimentos, também foi referida pelo diretor da Fundação de Hematologia e Hemoterapia da Bahia, que acrescentou referência aos pacientes com dengue (Fundação de Hematologia e Hemoterapia da Bahia - Hemoba, 29.04.20). A coordenadora da Fundação de Hematologia e Hemoterapia de Rondônia ainda "esclareceu que não é a Covid-19 quem dita a demanda pelo sangue tipos $\mathrm{O}+$ e $\mathrm{O}-$ no banco, muito menos, a redução do número de cirurgias e de acidentes no atual período de quarentena". Salientou que "esses tipos são os que mais transfundem" e fez destaque ao atendimento às necessidades urgentes dos pacientes com câncer (Fhemeron, 30.04.20).

Outros declarações identificadas nos sites trouxeram que a redução da capacidade de atendimento das unidades móveis (Centro de Hemoterapia e Hematologia de Goiás - Hemogo, 
Quadro 1. Consequências da pandemia, pela adoção de medidas de distanciamento social, sobre os serviços hemoterápicos do Brasil, abril, 2020.

\begin{tabular}{|l|l|}
\hline Consequências & Serviços Hemoterápicos - Hemorrede estadual \\
\hline $\begin{array}{l}\text { Redução nos níveis de estoque de sangue pela queda nas } \\
\text { doações }\end{array}$ & $\begin{array}{l}\text { Hemoal, 07.04.20; Hemorio, 07.04.20; } \\
\text { Hemoam, 01.04.20; Hemomar, 08.04.20 }\end{array}$ \\
\hline $\begin{array}{l}\text { Aumento do tempo de espera para o atendimento pela } \\
\text { redução de capacidade de atendimento das unidades móveis }\end{array}$ & Hemomar, 08.04.20; Hemogo, 24.04.20 \\
\hline $\begin{array}{l}\text { Alteração no funcionamento e organização dos serviços } \\
\text { pela adoção de medidas de precaução/segurança e } \\
\text { estabelecimento de novas rotinas }\end{array}$ & $\begin{array}{l}\text { A maioria registrou alguma mudança no } \\
\text { funcionamento e organização (Quadro 2) }\end{array}$ \\
\hline
\end{tabular}

Fonte: sites dos Hemocentros do Brasil, abril, 2020.

24.04.20) pela adoção de medidas para garantir maior segurança aos doadores, estendeu o tempo de espera para o atendimento e algumas pessoas não tem paciência ou não podem esperar (Hemomar, 08.04.20), influenciando, também, nos níveis dos estoques.

Demandas e mudanças na organização e funcionamento dos serviços hemoterápicos

O contexto da pandemia impactou sobre os níveis de estoque de sangue, de modo que estratégias para organização, funcionamento e rotina dos serviços precisaram ser implantadas e/ou implementadas. Este estudo revela que tais estratégias estão relacionadas às medidas de segurança, precaução e redução de risco para propagação do vírus nos serviços, incorporação de novas tecnologias, dentre outras (Quadro 2).

Medidas de proteção e precaução da transmissibilidade do vírus foram implementadas ao longo do mês de abril, por exemplo: intensificação da higienização das áreas e superfícies (Hemoba, 13.04.20; Fundação Hemocentro de Brasília - FHB, 08.04.20); recomendação do uso de máscaras pelos pacientes e doadores (Hemoba, 29.04.20); respeito a distância mínima entre os doadores; ampliação da quantidade de dispensadores de álcool em gel; uso de equipamentos de segurança (Hemoba, 29.04.20; FHB, 08.04.20; Centro de Hemoterapia e Hematologia do Pará - Hemopa, 13.04.20; Fundação Pró-Sangue Hemocentro de São Paulo - Pró-Sangue, 27.04.20).

Novas medidas para proteção às pessoas, pacientes e candidatos à doação foram incorporadas, por exemplo, "medir a temperatura de todos os pacientes que chegam à instituição" (Hemopa, 13.04.20), demandando com isso um fluxo de encaminhamento, de modo que "em caso de febre ou outros sintomas da Covid 19, as pesso- as são direcionados a outras unidades de saúde pública, inclusive com impedimento temporário de doação (Hemoam, 03.04.20). Por medida de proteção, outros fluxos foram alterados e rotinas estabelecidas, por exemplo, no Centro de Hematologia e Hemoterapia do Pará, as consultas médicas são prioridade para os casos emergenciais, de pacientes que precisam receber transfusões, plaquetas e Fator, e, ainda os portadores de anemia aplásica, suspensão de atendimentos como fisioterapia e agendamento para resgate de medicamentos (Hemopa, 13.04.20).

A ampliação de pontos/locais de doação foi reportada nos sites. O Centro de Hematologia e Hemoterapia do Ceará, decidiu pela oferta de novos locais para doação em pontos estratégicos do estado para continuar recebendo voluntários durante a pandemia Hemoce, 23.04.20), ainda que em muitas realidades a implantação das tendas externas (Hemomar, 08.04.20), como medida para reduzir aglomerações (FHB, 22.04.20) tenha reduzido a capacidade de coleta de tais unidades de coleta (Hemogo, 03.04.20; Hemogo, 24.04.20). Nesse sentido, o agendamento de doação (Hemoba, 13.04.20; FHB, 20.04.20; Hemogo, 03.04.20; Hemogo, 24.04.20; Pró-Sangue, 27.04.20; Centro de Hematologia e Hemoterapia de Sergipe - Hemose, 27.04.20), a doação programada (Centro de Hemoterapia e Hematologia do Mato Grosso do Sul - Hemosul, 30.04.20) de grupos de até dez pessoas (Hemose, 20.04.20; Centro de Hemoterapia e Hematologia de Tocantins - Hemoto, 24.04.20) e o chamamento de pessoas saudáveis (Hemoba, 29.04.20; FHB, 02.04.20), também têm sido estratégias utilizadas.

Portanto, o contexto da pandemia influenciou sobre as rotinas dos serviços, inclusive, com ampliação do horário de funcionamento e manutenção dos serviços aos sábados e/ou em feriados (FHB, 22.04.20; Hemosul, 30.04.20; Hemominas, 
Quadro 2. Medidas e rotinas implementadas pelos serviços hemoterápicos no contexto da pandemia de Covid-19, abril, 2020.

\begin{tabular}{|c|c|c|}
\hline Medidas e rotinas & Descrição & $\begin{array}{c}\text { Fontes/registros nos sites das } \\
\text { Hemorredes }\end{array}$ \\
\hline \multirow{4}{*}{$\begin{array}{l}\text { Medidas de proteção } \\
\text { (individual, coletiva) e } \\
\text { organização do espaço } \\
\text { físico para precaução } \\
\text { da propagação e } \\
\text { transmissibilidade do vírus }\end{array}$} & $\begin{array}{l}\text { Recomendação do uso de máscaras pelos } \\
\text { doadores e pacientes }\end{array}$ & Hemoba, 29.04.20 \\
\hline & $\begin{array}{l}\text { Medição da temperatura de todos que } \\
\text { chegam à instituição }\end{array}$ & $\begin{array}{l}\text { Hemopa, 13.04.20; Hemoam, } \\
\text { 03.04.20 }\end{array}$ \\
\hline & $\begin{array}{l}\text { Intensificação da higienização das áreas e } \\
\text { superfícies }\end{array}$ & Hemoba, 13.04.20; FHB, 08.04.20 \\
\hline & $\begin{array}{l}\text { Alteração do layout das salas de espera } \\
\text { com respeito a distância mínima entre os } \\
\text { doadores; ampliação da quantidade de } \\
\text { dispensadores de álcool em gel }\end{array}$ & $\begin{array}{l}\text { Hemoba, 29.04.20; FHB, 08.04.20; } \\
\text { Hemopa, 13.04.20; Pró-Sangue, } \\
\text { 27.04.20; } \\
\text { Hemoam, 01.04.20 }\end{array}$ \\
\hline $\begin{array}{l}\text { Ampliação de pontos/locais } \\
\text { de doação }\end{array}$ & $\begin{array}{l}\text { Oferta de novos locais para doação em } \\
\text { pontos estratégicos; implantação de } \\
\text { tendas externas }\end{array}$ & $\begin{array}{l}\text { Hemoce, 23.04.20; Hemomar, } \\
08.04 .20\end{array}$ \\
\hline $\begin{array}{l}\text { Agendamento e } \\
\text { chamamento para doação }\end{array}$ & $\begin{array}{l}\text { Agendamento individual e de grupos para } \\
\text { doação }\end{array}$ & $\begin{array}{l}\text { Hemoam, 01.04.20; } \\
\text { Hemoba, 13.04.20; FHB, 20.04.20; } \\
\text { Hemogo, 03.04.20; Hemogo, 24.04.20; } \\
\text { Pró-Sangue, 27.04.20; Hemose, } \\
\text { 27.04.20; Hemosul, 30.04.20; } \\
\text { Hemose, 20.04.20; Hemoto, 24.04.20; } \\
\text { Hemoba, 29.04.20; FHB, 02.04.20 }\end{array}$ \\
\hline $\begin{array}{l}\text { Alteração nos dias e } \\
\text { horários de funcionamento } \\
\text { dos serviços hemoterápicos }\end{array}$ & $\begin{array}{l}\text { Ampliação do horário de funcionamento } \\
\text { e manutenção dos serviços aos sábados e/ } \\
\text { ou em feriados }\end{array}$ & $\begin{array}{l}\text { FHB, 22.04.20; Hemosul, 30.04.20; } \\
\text { Hemominas, 15.04.20; Hemosc, } \\
\text { 01.04.20; Hemoto, 24.04.20 }\end{array}$ \\
\hline \multirow[t]{2}{*}{$\begin{array}{l}\text { Alteração na rotina de } \\
\text { oferta e fluxos de serviços }\end{array}$} & $\begin{array}{l}\text { Consultas médicas como prioridade } \\
\text { para os casos emergenciais; suspensão de } \\
\text { alguns atendimentos; agendamento para } \\
\text { dispensação de medicamentos }\end{array}$ & Hemopa, 13.04 .20 \\
\hline & $\begin{array}{l}\text { Validação e/ou oferta e realização de testes } \\
\text { diagnósticos para Covid-19 }\end{array}$ & $\begin{array}{l}\text { Hemoce, 14.04.20; Hemoce, 15.04.20; } \\
\text { Hemominas, 22.04.20 }\end{array}$ \\
\hline $\begin{array}{l}\text { Incorporação de } \\
\text { tecnologias de informação }\end{array}$ & $\begin{array}{l}\text { Incorporação de tecnologias de } \\
\text { informação para atendimento/ } \\
\text { agendamento, informações e } \\
\text { esclarecimento de dúvidas dos doadores }\end{array}$ & $\begin{array}{l}\text { Pró-Sangue, 01.04.20; Pró-Sangue, } \\
13.04 .20\end{array}$ \\
\hline $\begin{array}{l}\text { Estratégias novas e/ou } \\
\text { manutenção de medidas } \\
\text { variadas para estímulo à } \\
\text { doação }\end{array}$ & $\begin{array}{l}\text { Parcerias com serviços, instituições e } \\
\text { meios de comunicação; parcerias com } \\
\text { aplicativos de transporte; coleta em } \\
\text { condomínios; transporte gratuito para o } \\
\text { trajeto }\end{array}$ & $\begin{array}{l}\text { Hemopa, 30.04.20; Hemoba, 27.04.20; } \\
\text { Hemoal, 22.04.20; Hemoam, } \\
\text { 14.04.20; Hemoce, 04.04.20; Hemopi, } \\
\text { 06.04.20; Hemorio, 07.04.20; } \\
\text { Hemonorte, 20.04.20; Pró-Sangue, } \\
\text { 27.04.20; FHB, 08.04.20; FHB, } \\
\text { 20.04.20; Hemomar, 03.04.20; } \\
\text { Hemogo, 01.04.20; Hemogo, 29.04.20 }\end{array}$ \\
\hline $\begin{array}{l}\text { Oferta de cursos e eventos } \\
\text { relacionados às demandas } \\
\text { de enfrentamento da } \\
\text { pandemia }\end{array}$ & $\begin{array}{l}\text { Atualização dos critérios de triagem, } \\
\text { protocolos, procedimentos e treinamento } \\
\text { para trabalhadores de saúde }\end{array}$ & $\begin{array}{l}\text { Hemoam, 03.04.20; Hemogo, } \\
\text { 03.04.20; Hemogo, 24.04.20; } \\
\text { Hemominas, 22.04.20 }\end{array}$ \\
\hline
\end{tabular}

Fonte: sites das Hemorredes. As medidas citadas neste quadro foram identificadas a partir das publicações dos sites no mês de abril/2020, o que não exclui que outros serviços hemoterápicos também as tenham adotado.

15.04.20; Centro de Hemoterapia e Hematologia de Santa Catarina - Hemosc, 01.04.20; Hemoto, 24.04.20). Alguns Hemocentros incluíram na sua rotina, no mês de abril, a validação, a oferta e a realização de testes diagnósticos para Covid-19, por exemplo, o Centro de Hematologia e Hemo- 
terapia do Ceará validou a eficácia dos testes rápidos para Covid-19 (Hemoce, 15.04.20) e está habilitado para realizar o diagnóstico laboratorial de coronavírus, sendo o material analisado no Laboratório de Biologia Molecular do Hemoce (Hemoce, 14.04.20). E, também, desde o início do mês de abril, a Fundação Hemominas passou a integrar a Redelab Covid-19 e tem contribuído para o aumento da capacidade de realização dos testes no SUS (Hemominas, 22.04.20).

Tecnologias de informação têm sido incorporadas a fim de agilizar o atendimento e qualificar a experiência de doadores nos Hemocentros. A Fundação Pró-Sangue, em São Paulo, estabeleceu parceria com uma empresa especializada que utiliza Inteligência Artificial a fim de facilitar a busca de informações e esclarecimento de dúvidas dos doadores (Pró-Sangue, 01.04.20) e modernizou seu sistema de agendamento a partir da parceria com uma startup brasileira, passando a conferir maior agilidade e autonomia para os candidatos no agendamento ou cancelamento da sua doação (Pró-Sangue, 13.04.20).

A manutenção de velhas estratégias, como as parcerias para o incentivo a doação, foi referida nos sites visitados, entretanto, novos parceiros e novas estratégias também foram destacadas. Um processo de sanitização para higienizar e eliminar agentes causadores de várias infecções foi citado no Pará, mediante parceria com as prefeituras de dois municípios (Hemopa, 30.04.20). Na Bahia, foi publicada parceria com serviço de transporte para divulgação de informações e mensagem para a estímulo à doação (Hemoba, 27.04.20). A coleta externa em condomínios foi adotada pelo Hemorio e tem sido ampliada para outros estados. Para o deslocamento para as unidades de coleta, muitos estados têm estabelecido parcerias com aplicativos de transporte (Hemoam, 14.04.20; Hemoce, 04.04.20; Centro de Hematologia e Hemoterapia do Piauí - Hemopi, 06.04.20; Hemorio, 07.04.20; Centro de Hematologia e Hemoterapia do Rio Grande do Norte - Hemonorte, 20.04.20; Pró-Sangue, 27.04.20) e/ou disponibilizado transporte gratuito para o trajeto de ida e volta com número restrito de pessoas dentro do veículo (FHB, 08.04.20; FHB, 20.04.20; Hemomar, 03.04.20; Hemogo, 29.04.20). Também, conforme iniciativa do Tribunal de Justiça e Secretaria de Segurança Pública, a Hemorrede do Estado de Goiás aguarda incremento no número de doações, diante da Portaria 01/2020 da Vara de Execuções de Penas e Medidas Alternativas, que determina que a doação de sangue realizada nos meses de março a junho de 2020 equivalerá a
35 horas de prestação de serviços à comunidade pelos apenados (Hemogo, 01.04.20).

Os Hemocentros ainda têm promovido, em alguns casos articulados com outras instituições, qualificações, capacitações e treinamentos relacionados às demandas de enfrentamento da pandemia. Por exemplo, atualização dos critérios de triagem, treinamento sobre o uso racional de equipamento de proteção individual (EPI’s) para orientar sobre a utilização adequada (Hemoam, 03.04.20); sobre os protocolos de segurança (Hemogo, 03.04.20; Hemogo, 24.04.20); procedimentos e ajustamento das equipes (Hemominas, 22.04.20).

\section{Discussão}

Anterior a epidemia de Covid-19, os estoques de sangue variavam entre os estados do Brasil, no entanto, a partir de meado de março, algumas hemorredes anunciavam estoques de sangue, em especial dos grupos sanguíneos $\mathrm{O}$ e $\mathrm{AB}$, positivo e negativo, em níveis críticos, por exemplo, nos estados de Alagoas, Bahia, Mato Grosso do Sul, Espírito Santo, Maranhão, Rio Grande do Norte, Rondônia, Minas Gerais, Rio de Janeiro e São Paulo $^{11}$. A situação de (des)abastecimento seguiu nos Hemocentros do Brasil, durante o mês de abril, conforme demonstrado aqui, inclusive, justificada pela adoção e manutenção necessária de medidas de distanciamento social para conter a propagação do vírus SARS-Cov-2.

Dentre as medidas de distanciamento social ${ }^{6}$, a interrupção das atividades na comunidade e, especificamente, o fechamento de centros/unidades de doação em muitas áreas ao redor do mundo, impactaram na oferta de "recursos inestimáveis" à saúde ${ }^{4}$. Portanto, a redução significativa do número de doações de sangue é confirmada como consequência das restrições sociais feitas pelos governos para combater a disseminação de Covid-1912. Por exemplo, na cidade de Nova York foi registrada redução significativa do suprimento de sangue durante a semana de 16 de março de 2020 quando as escolas, empresas e instituições religiosas foram fechadas devido ao surto de coronavírus ${ }^{4}$.

$\mathrm{Na}$ China, o primeiro país que registrou casos de SARS-Cov-2, as rigorosas medidas de distanciamento social efetivamente continham o vírus, no entanto, restrições de mobilidade implementadas também tiveram um profundo impacto no sistema de saúde, incluindo redução na doação de sangue em muitas áreas do país, requerendo, 
portanto, iniciativas e estratégias para assegurar o suprimento de sangue ${ }^{1}$.

Nesse sentido, há que destacar que o distanciamento social e a consequência sobre a disponibilidade de sangue trouxeram a necessidade de reorganizar os serviços hemoterápicos e incluir novas estratégias e ações para assegurar o suprimento de sangue nos países, inclusive no Brasil, conforme revelou este estudo. Assim, medidas de segurança e precaução para os candidatos à doação, pacientes e trabalhadores, bem como, novas rotinas, fluxos e tecnologias foram incorporadas aos serviços, desde a adoção de protocolos, tecnologias de informação e comunicação, oferta de testes diagnósticos para Covid-19, e ações de estímulo a doação foram intensificadas juntos aos antigos e novos parceiros.

Desde o início dos primeiros casos de Covid-19 no Brasil, os Hemocentros incorporaram à sua rotina o agendamento de doações para assegurar o estoque de sangue e evitar aglomerações nos serviços, disponibilização de serviços de transporte para o deslocamento, ampliação dos ambientes de sala de espera, protocolos com orientações para disposição das cadeiras na espera e na sala de doação, uso de EPI e intensificação da limpeza ${ }^{11}$. Além disso, a epidemia também acrescentou novo perfil de usuários que adentram a sala de coleta, os quais devem ser triados em acordo com novos critérios estabelecidos e passíveis de atualização conforme o comportamento da pandemia. Portanto, durante esse período, a segurança de doadores e trabalhadores deve permanecer uma prioridade de qualquer serviço de coleta e transfusão, o que implica a adoção de um conjunto de medidas ${ }^{8}$, inclusive, a oferta de treinamento sobre o uso adequado de EPI e aplicação de precauções universais para a segurança dos trabalhadores ${ }^{9}$.

Estudos reportam as experiências de diversos países do mundo na pandemia e as medidas adotadas para manter e assegurar o sistema de coleta e fornecimento de sangue. As ações variam desde a sensibilização para estímulo à doação através de recursos informativos, mídia e comunicação $\mathrm{O}^{1,2,5}$ até a adoção de novos protocolos, fluxos e rotinas, tais como a triagem de doadores de sangue ${ }^{5}$, implementação de protocolos de falta e revisão de ordens de transfusão ${ }^{13}$, adoção de programa de gerenciamento de sangue do paciente (PBM) com utilização dos sistemas de informação ${ }^{2,14}$, abordagem centrada no paciente, aplicação de consensos de boas práticas ${ }^{12}$, mudança na dinâmica das atividades hospitalares que passam de operações rotineiras para pandêmicas e urgentes ${ }^{13}$, dentre outras.
Ainda no conjunto das estratégias para mitigar a escassez de sangue, campanhas de informação médica sobre os limiares apropriados de transfusão e uso apropriado de produtos farmacêuticos ${ }^{9}$, assim como, a organização na prestação de cuidados e dispensação de produtos sanguíneos a pacientes que dependem destes como recurso terapêutico, têm merecida atenção e sido incorporadas na rotina dos serviços nos países. Por exemplo, no Reino Unido, o aconselhamento nacional especializado a partir de um grupo de trabalho para a Covid-19 foi implantado para atualização médica, com orientações e conselhos práticos para o uso ideal de produtos sanguíneos e métodos para mitigar o uso de sangue nos pacientes com hemoglobinopatias ${ }^{15}$.

Uma Nota Técnica publicada pela Rede Covida, no Brasil, apresenta uma revisão de recomendações para o planejamento e a organização dos serviços hemoterápicos, tendo em vista a garantia do suprimento de sangue às necessidades do sistema de saúde e o fornecimento de plasma convalescente para os testes no tratamento de Covid-1916. A importância do planejamento e a organização dos serviços para dar respostas aos eventos foram apontadas como "lições aprendidas com esta pandemia", por representantes de bancos e departamentos de transfusão de diferentes países do mundo?

Para a discussão, cabe acrescentar que a disseminação do novo coronavírus também produziu o intenso debate sobre segurança do sangue, inclusive, com emissão de recomendações por autoridades sanitárias, enfatizando a não existência de evidências cientificamente documentadas sobre transmissão por transfusão de hemocomponentes $^{12}$. Por exemplo, na Itália, recomendações com medidas preventivas para a transmissão do SARS-CoV-2 foram publicadas e atualizadas em várias versões/decretos ${ }^{2}$. No Brasil, também foram publicadas notas técnicas que, informa os critérios para triagem clínica de dengue (DENV), chikungunya (CHIKV), zika (ZIKV) e coronavírus (SARS, MERS, 2019-nCoV) nos candidatos à doação de sangue $^{17} \mathrm{e}$, atualiza os critérios relacionados ao risco de infecção pelo SARS-CoV-2 ${ }^{18}$. Tais notas, portanto, esclarecem sobre critérios que geram inaptidão temporária para doação, que no caso brasileiro adiciona-se a ameaça do retorno às epidemias por arboviroses ${ }^{19}$ e apresentam orientações gerais para o funcionamento dos serviços hemoterápicos para evitar a propagação do vírus e proteger a saúde de pacientes, doadores e trabalhadores, o que em alguma medida repercute sobre o número de doações e as condições de estoque. 
Por fim, este estudo evidenciou que a adoção das medidas de distanciamento social demandou mudanças na rotina e organização do sistema de coleta e suprimento de sangue, entretanto, tais medidas, necessárias e efetivas, neste contexto de pandemia de crescimento ascendente no Brasil - registro de 431.609 casos confirmados em 28 de maio de 2020 (https://painel.covid19br.org/), devem levar em conta para sua flexibilização, o monitoramento da situação epidemiológica, a velocidade da transmissão da epidemia e o número de $\operatorname{casos}^{20}$. Assim, para o atendimento as demandas dos serviços de saúde no que tange aos produtos sanguíneos, a divulgação, o compartilhamento e a incorporação de estratégias, práticas e rotinas pelos Hemocentros devem estar em plena atividade nesse contexto de pandemia.

\section{Considerações finais}

Este estudo evidencia que a situação epidemiológica e as medidas necessárias para o seu controle influenciaram sobre a situação dos estoques e disponibilidade de sangue, e, demandaram, consequentemente, mudanças na organização dos serviços hemoterápicos.

Assim como em outros países, na realidade brasileira, a adoção das medidas de distanciamento tem exigido uma reorganização dos ser- viços hemoterápicos, de modo que estratégias, ações e rotinas têm sido incorporadas e/ou intensificadas para assegurar a proteção, mitigar os riscos de propagação do vírus e garantir o suprimento de sangue e hemocomponentes para o atendimento às necessidades do sistema de saúde.

Portanto, as estratégias e as novas rotinas incorporadas aos serviços, discutidas neste artigo à luz de experiências de outros países, são importantes de serem mantidas e implementadas a fim de conter a situação epidemiológica de pandemia pela Covid-19 e mitigar os riscos à saúde da população.

\section{Agradecimentos}

Agradeço o apoio na coleta e atualização da matriz de notícias das Hemorredes através do Observatório de Análise Política em Saúde do Instituto de Saúde Coletiva (OAPS/ISC/UFBA) e o apoio científico da Rede CoVida - Ciência, Informação e Solidariedade (Cidacs/Fiocruz e UFBA). 


\section{Referências}

1. Cai X, Ren H, Chen F, Li L, Lei H, Wang X. Blood transfusion during the Covid-19 out break. Blood Transfus 2020; 18(2):79-82.

2. Franchini M, Farrugia A, Velati C, Zanetti A, Romanò L, Grazzini G, Lopez N, Pati I, Marano G, Pupella S, Liumbruno GM. The impact of the SARS-Cov-2 outbreak on the safety and availability of blood transfusions in Italy. Vox Sang 2020; 10.1111/vox.12928.

3. Kwon SY, Kim EJ, Jung YS, Jang JS, Cho NS. Post-Donation Covid-19 Identification in Blood Donors. Vox Sang 2020; 10.1111/vox.12925.

4. Shander A, Goobie SM, Warne MA, Aapro M, Bisbe E, Perez-Calatayud AA, Callum J, Cushing MM, Dyer WB, Erhard J, Faraoni D, Farmer S, Fedorova T, Frank SM, Froessler B, Gombotz H, Gross I, Guinn NR, Haas T, Hamdorf J, Isbister JP, Javidroozi M, Ji H, Kim YW, Kor DJ, Kurz J, Lasocki S, Leahy MF, Lee CK, Lee JJ, Louw V, Meier J, Mezzacasa A, Munoz M, Ozawa S, Pavesi M, Shander N, Spahn DR, Spiess BD, Thomson J, Trentino K, Zenger C, Hofmann A, International Foundation of Patient Blood Management (IFPBM) and Society for the Advancement of Blood Management (SABM) Work Group. The essential role of patient blood management in a pandemic: a call for action. Anesth Analg 2020; 131(1):74-85.

5. Raturi M, Kusum A. The active role of a blood center in out pacing the transfusion transmission of $\mathrm{Co}-$ vid-19. Transfus Clin Biol 2020; 27(2):96-97.

6. Wilder-Smith A, Freedman DO. Isolation, quarantine, social distancing and community containment: pivotal role for old-style public health measures in the novel coronavirus (2019-nCoV) outbreak. J Travel Med 2020; 27(2):taaa020

7. Garraud O. Covid-19: is a paradigm change to be expected in health care and transfusion medicine? Transfus Clin Biol 2020; 27(2):59-60.

8. Raturi M. A Quick "Can I Donate Blood” Self-Assessment Tool Amid the Covid-19 Outbreak. Transfus Clin Biol 2020; 27(2):169-170.

9. Yazer MH, Jackson B, Pagano M, Rahimi-Levene N, Pares V, Bueno JL, Jackson RP, Shan H, Amorim-Filho L, Lopes M-E, Boquimpani C, Sprogøe U, Bruun MT, Titlestad K, Rushford K, Wood EM, McQuilten ZK, Angelis V, Donne MD, Murphy M, Staves J, Cho D, Nakamura F, Hangaishi A, Callum J, Lin Y, Mogaddam M, Gharehbaghian A, Lozano M, Vox Sanguinis International Forum on Transfusion Services About Response to Covid-19. Vox Sang 2020; 115(6):536-542.

10. Fan BE, Ong KH, Chan SSW, Young BE, Chong VCL, Chen SPC, Lim SP, Lim GP, Kuperan P. Blood and blood product use during covid-19 infection. Am J Hematol 2020; 95(7):E158-E160.

11. Souza MKB, Lima YOR, Cavalcante LLR. (Des)Abastecimento do estoque de sangue e estratégias para o aumento da doação em tempos de Covid-19 [Internet]. 2020 [acessado 2020 Abr 18]. Disponível em: https:// www.analisepoliticaemsaude.org/oaps/pensamentos/551b2b2b673ef8efe72170ac42f1e8ba/7/
12. Baron DM, Franchini M, Goobie SM, Javidroozi M Klein AA, Lasocki S, Liumbruno GM, Muñoz M, Shander A, Spahn DR, Zacharowski K, Meybohm P. Patient Blood Management during the Covid-19 Pandemic - A Narrative Review. Anaesthesia 2020; 75(8):1105-1113.

13. Pagano MB, Hess JR, Tsang HC, Staley E, Gernsheimer T, Sen N, Clark C, Nester T, Bailey C, Alcorn K. Prepare to adapt: blood supply and transfusion support during the first 2 weeks of the 2019 novel coronavirus (COVID-19) pandemic affecting Washington state. Transfusion 2020; 60(5):908-911.

14. Mascaretti L, De Angelis V, Berti P. The severe acute respiratory syndrome coronavirus 2 (SARS-Cov-2) pandemic and transfusion medicine: reflections from Italy. Blood Transfus 2020; 18(2):77-78.

15. Roy NBA, Telfer P, Eleftheriou P, De la Fuente J, Drasar E, Farrukh S, Roberts D, Atoyebi W, Trompeter S, Layton DM, Lugthart S, Stuart-Smith S, Chakravorty S, Wright J, Porter J, Inusa B, Howard J, National Haemoglobinopathy Panel. Protecting Vulnerable Patients With Inherited Anaemias From Unnecessary Death During The Covid-19 Pandemic. Br J Haematol 2020; 189(4):635-639.

16. Souza MKB. Nota Técnica 05/2020. Revisão sobre recomendações para o planejamento e a organização dos serviços hemoterápicos durante a pandemia pelo SARS-COV-2 [Internet]. [acessado 2020 Maio 25]. Disponível em: https://covid19br.org/main-site-covida/ wp-content/uploads/2020/05/Nota-Tecnica-05-hemoterapicos.pdf

17. Brasil. Ministério da Saúde (MS). Nota técnica $n^{o}$ 5/2020-CGSH/DAET/SAES/MS. Atualização dos critérios técnicos para triagem clínica de dengue (DENV), chikungunya (CHIKV), zika (ZIKV) e coronavírus (SARS, MERS, 2019-nCov) nos candidatos à doação de sangue [Internet]. 2020 [acessado 2020 Mar 28]. Disponível em: https://www.saude.gov.br/images/ pdf/2020/fevereiro/21/SEI-MS---0013484477---Nota-T--cnica.pdf

18. Brasil. Ministério da Saúde (MS). Secretaria de Atenção Especializada à Saúde. Departamento de Atenção Especializada e Temática Coordenação-Geral de Sangue e Hemoderivados. Nota Técnica No 13/2020CGSH/DAET/SAES/MS. Atualização dos critérios técnicos contidos na Nota Técnica No 5/2020-CGSH/ DAET/SAES/MS para triagem clínica dos candidatos à doação de sangue relacionados ao risco de infecção pelo SARS-CoV-2 [Internet]. 2020 [acessado 2020 Abr 26]. Disponível em: https://www.saude.gov.br/images/ pdf/2020/marco/27/SEI-MS---0014052636---Nota-T--cnica---n---13-2020.pdf

19. Cioffi JGM. Viroses, transfusões de sangue e doações voluntárias [Internet]. 2016 [acessado 2020 Mar 26]. Disponível em: http://blog.saude.mg.gov. br/2016/06/06/artigo-viroses-transfusoes-de-sangue -e-doacoes-voluntarias/ 
20. Aquino EML, Silveira IH, Pescarini JM, Aquino R, Souza-Filho JÁ, Rocha AS, Ferreira A, Victor A, Teixeira C, Machado DB, Paixão E, Alves FJO, Pileco F, Menezes G, Gabrielli L, Leite L, Almeida MCC, Ortelan N, Fernandes QHRF, Ortiz RJF, Palmeira RN, Pinto Junior EP, Aragão E, Souza LEPF, Barral Netto M, Teixeira MG, Barreto ML, Ichihara MY, Lima RTRS. Medidas de distanciamento social no controle da pandemia de COVID-19: potenciais impactos e desafios no Brasil. Cien Saude Colet 2020; 25(Supl. 1):2423-2446.

Artigo apresentado em 28/05/2020

Aprovado em 07/10/2020

Versão final apresentada em 09/10/2020 